\title{
THE PIECEWISE SOLUTION OF A CERTAIN CLASS OF TRANSPORTATION PROBLEMS
}

\author{
(Presented by I. Opik)
}

This paper considers a special class of transportation problems. Its formulation is about the same as the well-known Hitchcock problem, with an additional constraint that all (or some) of the variables are binary. The transportation network consists of $m$ sources, each producing a finite amount $a_{i}$ of material, and $n$ users, whose demands $d_{j}$ of that material are known; a cost $c_{i j}$ of the material transportation per unit is given on each arc $i j$ leading from source $i$ to user $j$; and the flow of material that minimizes the total transportation cost is to be determined, taking into account the constraint that a user is allowed to be supplied by one source (or a group of sources) only, so that the amount of material transported from a source (or a group of sources) to a user can be equal either to zero or to the demand of the user. It is also supposed that $m$ is much less than $n$. The same problem was considered by A. De Maio and C. Roveda in $\left[{ }^{1}\right]$.

The paper proposes a method for the piecewise solution of the problem. The method is based on partitioning of the transportation network into smaller isolated subnetworks and solving the problem of a smaller scale for each subnetwork. The method is an iterative one and it gives an optimal solution to the traditional transportation problem and an approximate one to the problem with binary variables. The idea of the partitioning of the network is taken from Kron's «diacoptics» $\left[{ }^{2}\right]$. There are also used some elements from Klein's algorithm for minimal cost flow [ $\left.{ }^{3}\right]$.

An application of the model to a real practical problem is described at the end of the paper.

\section{The mathematical model}

Define: $M=\{1, \ldots, m\}, N=\{1, \ldots, n\}$ and $M^{\prime}=\left\{1, \ldots, m^{\prime}\right\}$, where $0 \leqslant m^{\prime} \leqslant m$. The problem is to find $X$ minimizing

$$
z=\sum_{i \in M} \sum_{j \in N} c_{i j} d_{j} x_{i j}
$$

subject to

$$
\sum_{j \in N} d_{j} x_{i j} \leqslant a_{i} \quad(\forall i \in M)
$$




$$
\begin{array}{cc}
\sum_{i \in M} x_{i j}=1 & (\forall j \in N), \\
0 \leqslant x_{i j} \leqslant 1 & (\forall i \in M, \forall j \in N), \\
x_{i j}=\left\{\begin{array}{l}
1 \\
0
\end{array} \quad\left(\forall i \in M^{\prime}, \forall j \in N\right) .\right.
\end{array}
$$

Firstly we shall consider the case of $m^{\prime}=0$, when the problem (1)-(5) becomes the linear programming (LP) transportation problem (1)-(4), and then integer (when $m^{\prime}=m$ ) and mixed integer (when $0<m^{\prime}<m$ ) problems.

Suppose, without loss of generality, that the largest flows $b_{i j}$ allowed over the arcs $i j$ are equal to $d_{j}$ and $\min _{i \in M}\left\{a_{i}\right\} \geqslant \max _{j \in N}\left\{d_{j}\right\}$. For the LP transportation problem $b_{i j}=\min \left(a_{i}, d_{j}\right)$. In all of the three cases divide the transportation network $Q$ into $m$ isolated subnetworks $Q_{i}$ so that each one consists of one source $i$ and some number of users $j$ connected by arcs with the source. The rest of the arcs and all the sources $i \in M$, and the users $j \in N$ form a subnetwork $Q^{\pi}$. The same partitioning Kron has proposed in «diacoptics».

Definition. Let us call an n-cycle a negative cycle consisting of $\operatorname{arcs} p s \in Q^{\mathrm{I}}, s q \in Q_{q}, q r \in Q^{\mathrm{m}}$ and $r p \in Q_{p}$, where nodes $p, q$ are sources and $r, s$ are users $\left(r \in Q_{p}, s \in Q_{q}\right)$.

\section{The optimal Algorithm I for a piecewise solution of the LP transportation problem}

We now outline Algorithm I.

Initial Iteration. S tep 1. Define $m(m-1)$ vectors $\mathrm{f}^{(i g)}$ with elements

$$
f_{j}^{(i g)}=c_{i j}-c_{g j} \quad\left(\forall j \in Q_{g} ; \forall i, g \in M, i \neq g\right) .
$$

Here $j$ is not an ordinal number of element but it is the number of user from subnetwork $Q_{g}$. Go to the next step.

Ste p 2. Suppose that the flows over the $\operatorname{arcs} i j \in Q_{i}$ are equal to $b_{i j}$ and determine an $n$-cycle of minimal cost. Find

$$
\psi=f_{\eta}^{(q p)}+f_{\mu}^{(p q)}=\min _{i, g \in M, i \neq g}\left(\min _{r \in Q_{o}}\left\{f_{r}^{(i g)}\right\}+\min _{s \in Q_{t}}\left\{f_{s}^{(g i)}\right\}\right) .
$$

If $\psi \geqslant 0$, no $n$-cycle can exist. Go to Step 4. If $\psi<0$, go to the next step.

Step 3. Change the partitioning of the transportation network $Q$ so that to eliminate the possibility of the existence of the $n$-cycle found at Step 2. The $n$-cycle consists of the arcs $p \mu, \mu q, q \eta$ and $\eta p\left(\mu \in Q_{q}\right.$, $\left.\eta \in Q_{p}\right)$. Form new subnetworks $Q_{p}$ and $Q_{q}$ :

$$
Q_{p}:=Q_{p} \cup \mu / \eta, \quad Q_{q}:=Q_{q} \cup \eta / \mu .
$$

(8) is just changing the places of the users $\eta$ and $\mu$. Remove all the elements $f_{\eta}^{(i p)}(\forall i \neq p)$ and $f_{\mu}^{(i q)}(\forall i \neq q)$ from further consideration and determine new elements $f_{\mu}^{(i p)}$ and $f_{\eta}^{(i q)}$ according to (6). Go back to Step 2. 
Step 4. Find a feasible solution $\left\{x_{i j}^{(0)}\right\}$ to problem (1)-(4). Define a set $Q^{0}$ of subnetworks $Q_{v}^{0}$ for which an inequality

$$
a_{i} \leqslant \sum_{j \in Q_{t}} d_{j}
$$

holds. The rest of subnetworks form a set $Q^{*}$.

For each subnetwork $Q_{v}^{0} \in Q^{0}$ solve a trivial problem of minimizing

$$
\bar{z}_{v}=\sum_{j \in Q_{v}{ }^{\circ}} c_{v j} d_{j} x_{v j}
$$

subject to

$$
\begin{gathered}
\sum_{j \in Q_{v}{ }^{\circ}} d_{j} x_{v j}=a_{v}, \\
0 \leqslant x_{v j} \leqslant 1 \quad\left(\forall j \in Q_{v}^{0}\right) .
\end{gathered}
$$

Satisfy all the user's demands for each $Q_{w}^{*} \in Q^{*}$ and compute $\bar{z}_{10}=$ $=\sum_{j \in Q_{w^{*}}} c_{w j} d_{j} \quad\left(\forall w \in Q^{*} \cap M\right)$. Compute amounts $a_{w}^{*}$ of the rest of the material in the sources $w: a_{w}^{*}=a_{w}-\underset{j \in Q_{w}^{*}}{\sum d_{j}}\left(\forall w \in Q^{*} \cap M\right)$.

There still remains a set $D$ of users $r \in D \subset Q^{0}$ whose demands $d^{*}{ }_{r}$ are not completely satisfied $\left(d_{r}^{*} \neq d_{r}\right.$ for not more than $m-1$ users $)$. Consider a part of the subnetwork $Q^{\text {m }}$ which consists of sources $w \in Q^{*}$ and users $r \in D$ connected by arcs $w$. Now we have to solve a transportation problem of a smaller size, using the computed values $a_{w}^{*}$ and $d_{r}^{*}$. If the problem is still of a large scale, we use again the same Algorithm I.

Compute the total transportation $\cos t z^{(0)}$. Let $\tau_{i}$ be subsidiary variables all equal to zero $(\forall i \in M)$. Go to the First Iteration which consists of the same following steps as any

$k$-Iteration $(k \geqslant 1)$. S te p 5 . Change the partitioning of the transportation network $Q$ so that

$$
Q_{i}=\left\{j: x_{i j}^{(k-1)}>0\right\} \quad(\forall i \in M) .
$$

Now $n \leqslant \sum_{i \in M} n_{i} \leqslant n+m \rightarrow 1$, where $n_{i}$ is the number of users in subnetwork $Q_{i}$ formed according to (13). Determine elements $f_{j}^{(i g)}$ as it is described in (6). Go to the next step.

Step 6. Test $\left\{\tau_{i}\right\}$ for the existence of a zero element. If there is none, the feasible solution $\left\{\begin{array}{c}x^{(h-1)} \\ i j\end{array}\right\}$ is an optimal solution and $z_{\text {opt }}=z^{(k-1)}$. If there is at least one $\tau_{i}=0$, go to the next step.

S te $\mathrm{p}$ 7. Find the sources $p$ and $q\left(p \neq q, \tau_{p}=0\right)$ and the user $r \in Q_{q}$ such that

$$
f_{r}^{(p q)}=\min _{j \in Q_{g}}\left\{f_{j}^{(i g)}\right\}
$$

for each $i \neq g ; i, g \in M$, where $\tau_{g}=0$. Go to the next step.

S te $\mathrm{p}$. Let $p:=q$, where $q$ is found by the previous operation. Find 


$$
f_{r}^{(p q)}=\min _{j \in Q_{t}}\left\{f_{j}^{(p i)}\right\} \quad(\forall i \in M, i \neq p) .
$$

If $q$ is equal to one of the $p$ 's which was found before, go to the next step. If $q$ is not equal to any $p$, go to Step 7 .

Step 9. Trace the cycle and compute its cost $\psi$ as a sum of the values of $f_{r}^{(p q)}$ starting from the $p$ which is equal to the last found $q$.

If $\psi \geqslant 0$ let $\tau_{p}$ be equal to 1 , where $p$ is found by $(14), z^{(k)}=z^{(k-1)}$ and $\left\{x_{i j}^{(k)}\right\}=\left\{x_{i j}^{(k-1)}\right\}$. Go to the $(k+1)$-Iteration. If $\psi<0$, determine $\delta=$ $=\min \left\{x_{q r}^{(k-1)}\right\}$ for each $q$ and $r \in Q_{q}$ of the negative cycle. Compute new flows over the arcs of the negative cycle:

$$
\begin{aligned}
& x_{p r}^{(k)}=x_{p r}^{(k-1)}+\delta, \\
& x_{q r}^{(k)}=x_{q r}^{(k-1)}-\delta
\end{aligned}
$$

for each $p, q$ and $r \in Q_{q}$ of the negative cycle. For the rest of the arcs $x_{i j}^{(k)}=x_{i j}^{(k-1)}$. Compute $z^{(k)}=z^{(k-1)}+\psi \delta$. Go to the $(k+1)$-Iteration.

Similar algorithm is described in $\left[{ }^{4}\right]$.

\section{Proof of Algorithm I}

It is obvious that we have to consider only one direction $p \rightarrow s \rightarrow q \rightarrow r \rightarrow p$ $\left(r \in Q_{p}, s \in Q_{q}\right)$ of the $n$-cycle (see the Definition). We have supposed (Step 2) that the flows over the $\operatorname{arcs} i j \in Q_{i}(\forall i \in M)$ were equal to $b_{i j}$ and, according to the Klein's rule $\left.{ }^{3}\right]$, the modified costs $c_{i j}{ }^{*}=\infty$ which eliminates the second direction of the $n$-cycle.

Theorem 1. If a node $r$ is excluded from a subnetwork $Q_{p}$ by performing of the operations (7) and (8), it will not be a part of the subnetwork $Q_{p}$, while further performing of the same operations.

The proof of Theorem 1 is simple but rather space consuming, and so we omit it in the present paper.

We can see now that performing of Steps 2 and 3 is a finite process (because $m$ is a finite number).

Theorem 2. Let $X=\left\{x_{i j}\right\}$ be a vector satisfying (2), (3) and (4) of a transportation problem. Define index sets $J_{i}=\left\{j: x_{i j}>0\right\}$. If $f_{j}^{(i g)}=$ $=c_{i j}-c_{g j}$ the necessary condition for the optimality of the solution $X$ is

$$
f_{r}^{(p q)} \leqslant f_{s}^{(p q)}
$$

for all $p$ and $q$ and all $r \in J_{p}$ and $s \in J_{q}(p \neq q ; p, q=1, \ldots, m)$.

$\mathrm{Proof}$. In fact the definition of the index sets $J_{i}$ coincides with the one of the subnetworks $Q_{i}$ (see Step 5 ). We know that any $n$-cycle of the network $Q$ divided into $m$ subnetworks $Q_{i}$ consists of some four nodes $p, s, q, r$, where $r \in Q_{p}$ and $s \in Q_{q}$. The cost of the $n$-cycle is equal to $c_{p s}+c_{s q}+c_{q r}+c_{r p}$, or using Klein's rule,

$$
\left(c_{p s}-c_{q s}\right)+\left(c_{q r}-c_{p r}\right)=f_{s}^{(p q)}-f_{r}^{(p q)} .
$$

Hence, $f_{s}^{(p q)}-f_{r}^{(p q)}<0$ means that there exists an $n$-cycle which includes some $r \in Q_{p}$ and $s \in Q_{q}$. But according to the well-known 
result, a flow in a network is optimal if, and only if, there are no negative cycles. Therefore, the solution $X$ is optimal if $f_{s}^{(p q)}-f_{r}^{(p q)} \geqslant 0$, or $f_{r}^{(p q)} \leqslant f_{s}^{(p q)}$ for all $r \in Q_{p}$ and $s \in Q_{q}$. Q.E.D.

The same results have been obtained by D. L. Adolphson and G. N. Thomas $\left[{ }^{5}\right]$ for the necessary and sufficient condition of an optimality of a $2 \times n$ transportation problem solution.

It can be easily proved that a negative cycle of a minimal cost has to include arcs determined by the operations (15) and/or (14), and it is obvious that the elimination of such cycle leads to an optimum more rapidly than elimination of any other negative cycle.

Starting from the First Iteration we have to consider $m-1$ users in two subnetworks because the flows over the arcs connecting these users with sources are less than $b_{i j}$. Hence, the transportation costs of these arcs are finite values for both directions. Algorithm I is not to be changed if the number of such users is less than $m-1$.

It is obvious that elimination of all negative cycles in a finite transportation network will be achieved by a finite number of iterations. The algorithm needs $m$ additional iterations for the control of the optimality. We use the subsidiary variables $\tau_{i}$ to prevent returning to the considered cycles. $\tau_{i}$ is equal to 1 if the cycle which «starts» from source $i$ has been examined, and $\tau_{i}=0$ otherwise.

Theorem 3. Let a transportation network $Q$ be divided into $m$ subnetworks $Q_{i}$ so that $Q_{i}=\left\{j: x_{i j}>0\right\}$, where $X=\left\{x_{i j}\right\}$ is a feasible solution of a transportation problem. Then the solution $X$ is optimal if, and only if,

$$
\sum_{i=\mu}^{v}\left(c_{p^{(t-1)} r^{(t)}}-c_{\left.p^{(t)} r^{(t)}\right)} \geqslant 0\right.
$$

for all $p^{(i-1)} \neq p^{(i)}, \quad r^{(i)} \in Q_{p^{(t)}}$ and where $p^{(\mu-1)}=p^{(v)}$.

Proof. The proof derives trivially from the mentioned theorem of non-existence of any negative cycles in an optimal flow.

In fact Theorem 2 is a particular case of Theorem 3.

\section{The approximate Algorithm II for a piecewise solution of a transportation problem with zero-one variables}

Now we outline Algorithm II.

Initial Iteration. Step 1. Define $m(m-1)$ vectors $\mathbf{f}^{(i g)}$ according to the rule $(6)$ and go to the next iteration.

$k$-Iteration $(k \geqslant 1)$. Steps 2 and 3 coincide with the same of Algorithm I.

Step 4. Find a feasible solution $\left\{x_{i j}^{(k)}\right\}$ of problem $(1)-(5)$. Define the sets $Q^{0}$ and $Q^{*}$ as it was done in Algorithm I. For each subnetwork $Q_{v}^{0} \in Q^{0}$ solve a problem similar to the knapsack problem. For each $Q_{w}^{*} \in Q^{*}$ compute $\bar{z}_{w}=\sum_{j \in Q_{10}} c_{w j} d_{j}$ and $a_{w}^{*}=a_{w}-\sum_{j \in Q_{w^{*}}} d_{j}$.

Solve a problem identical to the original problem (1)-(5) using the computed values $a_{w}{ }^{*}$ and non-satisfied demands $d_{r}(r \in D)$ with corresponding transportation costs $c_{w r}$. A scale of the latter problem is smaller than the one of the original problem because the number of $w$ 's 
is less than $m\left(m=|M|>\left|Q^{*} \cap M\right|\right)$ and the number of ' $r$ 's seems to be much less than $n(n=|N| \gg|D|)$.

Compute the total transportation cost $z^{(k)}$. Go to the next step.

S tep 5. Change the pantitioning of the network $Q$ so that

$$
Q_{i}=\left\{j: x_{i j}^{(k)}=1\right\} \text {. }
$$

Obviously, all the users $r \in D$ are to change their subnetworks. Exclude elements of the vectors $f^{(i g)}$ corresponding to each node $r \in D$ in previous partitioning and determine new elements $f_{r}^{(i g)}$ for all $r \in D ; i, g \in M$; $i \neq g$. Now $Q_{i} \cap Q_{g}=\varnothing(\forall i, g \in M, i \neq g)$. Go to the next step.

Ste p 6 . Find an $n$-cycle of a minimal cost $\psi$ using the rule (7). If $\psi \geqslant 0$, go to Step 8; otherwise go to the next step.

S te p 7. Let the cycle $p \rightarrow \mu \rightarrow q \rightarrow \eta \rightarrow p$ be found at the previous step $\left(\eta \in Q_{p}, \mu \in Q_{q}\right)$. Test two following inequalities

$$
\begin{aligned}
& \sum_{j \in Q_{p}} d_{j}-d_{\eta}+d_{\mu} \leqslant a_{p}, \\
& \sum_{j \in Q_{q}} d_{j}-d_{\mu}+d_{\eta} \leqslant a_{q}
\end{aligned}
$$

for holding. If both (17) and (18) hold, form new subnetworks $Q_{p}$ and $Q_{q}$ according to the rule (8). Change the elements of the vectors $f^{(i g)}$ which correspond to the users $\eta$ and $\mu$. Compute the total transportation cost

$$
\begin{aligned}
& z^{(k)}:=z^{(k)}+d_{\eta}\left(c_{q \eta}-c_{p \eta}\right)+d_{\mu}\left(c_{p \mu}-c_{q \mu}\right) \\
& x_{p \eta}^{(k)}=0, \quad x_{p \mu}^{(k)}=1, x_{q \mu}^{(k)}=0 \text { and } x_{q \eta}^{(k)}=1 . \text { Go to Step } 6 .
\end{aligned}
$$

If at least one inequality does not hold, exclude the latter $n$-cycle from further consideration and go back to Step 6 .

Step 8. If starting from a $k$-Iteration $(k \geqslant 2) \quad z^{(k)}>z^{(k-1)}$, stop performing of the algorithm and accept a solution $\left\{x_{i j}^{(k-1)}\right\}$ as an approximate solution to the problem $(1)-(5)$. If $z^{(k)} \leqslant z^{(k-1)}$, go to the next iteration.

Algorithm III for approximate solution to a mixed integer problem (1) - (5) is a combination of Algorithms I and II.

First attempt of solving this problem by decomposition of a network was made by the author in $\left[{ }^{6}\right]$.

\section{An application of the model and some concluding remarks}

The real problem that originated this research is the problem of projecting the fuel (or energy) distribution among users of some region. A number of sources in this problem practically does not exceed 10 or 15 , while a number of users can be about $10^{4}$. Some types of fuel (or energy) can be consumed in the same mountings (for example, all sorts of coal), but mostly each type needs its own equipment. So it cannot be planned to use both gas and coal (or petroleum and electricity) in the same place simultaneously.

The algorithms have been programmed in FORTRAN IV version for DOS/ES. Some practical problems with up to ten thousand users have been solved. The author has also developed an algorithm for solving a 
transportation problem with arc-capacity restriction by the same partitioning method.

It is worth noting that Algorithm I yields an optimal solution more rapidly if we use the partitioning into $m$ isolated subnetworks $Q_{i}$ in such a way that $Q_{p}=\varnothing$, where $a_{p}=\max \left\{a_{i}\right\} \quad(\forall i \in M)$, and numbers of users in the rest of the subnetworks are approximately $n a_{i} / \sum_{i=1}^{m} a_{i}$. Then according to (9), all the subnetworks $Q_{1}, \ldots, Q_{p-1}, Q_{p+1}, \ldots, Q_{m}$ get into the set $Q^{0}$, and at Step 4 for these subnetworks we solve a problem of minimizing

$$
\bar{z}_{v}=\sum_{j \in Q_{v}{ }^{\circ}}\left(c_{v j}-c_{p j}\right) d_{j} x_{v j},
$$

subject to (11) and (12).

So there is no need to solve a transportation problem, and we just have to compute $\hat{z}=\sum_{r \in D} c_{p r} d_{r}^{*}$.

The algorithms do not need a high amount of storage because we have to remember practically only the matrix $\left\|c_{i j}\right\|$ and vectors $\left\|a_{i}\right\|$ and $\left\|d_{j}\right\|$. In fact we can manage without vectors $f^{(i g)}$, but, of course, these vectors help to obtain an optimum in a shorter time.

\section{REFERENCES}

1. De Mai o, A., Roveda, C., Oper. Res., 19, № 6, 1406-1418 (1971).

2. Крон Г., Исследование сложных систем по частям - диакоптика, М., «Наука», 1971.

3. Kl e i n, M., Manag. Sci., 14, № 3, 205-220 (1967),

4. К о р че м к и н М., Изв. АН ЭССР, Обществ. науки, 27, № 3, 243-248 (1978).

5. Adolphson, D. L., Thom a s, G. N., SIAM J. Comput., 6, № 3, 481-486 (1977).

6. Корчемкин М. Б., В кн.: Модели и методы анализа экономических целенаправленных систем, Новосибирск, «Наука», 1977, с. $47-51$.

Academy of Sciences of the Estonian SSR, Institute of Economics

Received June 8, 1979

\section{KORTSEMKIN}

\section{TEATUD TUUPI TRANSPORDIULESANNETE OSITI LAHENDAMINE}

Artiklis on kirjeldatud transpordiülesannet, kus on vaja leida $\left\{x_{i j}\right\}$, mis minimeerib $z=\sum \sum c_{i j} d_{j} x_{i j} \quad$ tingimustel: $\quad \sum d_{j} x_{i j} \leqslant a_{i}(\forall i \in M) ; \quad \sum x_{i j}=1(\forall j \in N)$; $0 \leqslant x_{i j} \leqslant 1 \quad(\forall i \in M, \quad \forall j \in N) ; \quad x_{i j}=0 \bigvee 1 \quad\left(\forall i \in M^{\prime} \subset M, \quad \forall j \in N\right) . \quad$ Olesandes eeldatakse, et ressursside arv $|M|$ on tunduvalt väiksem tarbijate arvust $|N|$. Esitatud meetod sisaldab elemente G. Kroni diacoptica'st ja pōhineb transpordivōrgu jaotamisel $|M|$ ühisosata allvõrguks. Itereerimisel otsitakse lubatavat plaani ja ühtlasi elimineeritakse negatiivsed tsüklid. Selleks on lahendatud igal allvōrgul mõôtmetelt väiksemaid ülesandeid. On esitatud kaks algoritmi: algoritm I lineaarse programmeerimise transpordiülesannete lahendamiseks juhul, kui $M^{\prime}=\varnothing$, ja algoritm II diskreetsete ülesannete lahendamiseks, kui $M^{\prime} \equiv M$. On antud püstitatud ülesande majanduslik interpretatsioon. 


\section{M. КОРЧЕМКИН}

\section{РЕШЕНИЕ ПО ЧАСТЯМ ОДНОГО КЛАССА ТРАНСПОРТНЫХ ЗАДАЧ}

В статье рассматривается транспортная задача следующего типа: $z=$ $=\sum_{i \in M} \sum_{j \in N} c_{i j} d_{j} x_{i j} \rightarrow \min ; \sum_{j \in N} d_{j} x_{i j} \leqslant a_{i} \quad(\forall i \in M) ; \sum_{i \in M} x_{i j}=1 \quad(\forall j \in N) ; \quad 0 \leqslant x_{i j} \leqslant 1$ $(\forall i \in M, \quad \forall j \in N) ; \quad x_{i j}=0 \bigvee 1 \quad\left(\forall i \in M^{\prime} \subset M, \forall j \in N\right)$. Предполагается, $|M| \ll|N|$ и задача имеет решение. Предлагаемый метод решения такой задачи в своей основе содержит некоторые элементы «диакоптики» Крона и заключается в предварительном расчленении транспортной сети, иллюстрирующей данную задачу, на $|M|$ непересекающихся подсетей и последующем итеративном построении допустимого решения с устранением циклов отрицательной стоимости. При построении допустимого решения рассматривается ряд задач, меньших по сравнению с первоначальной размерностей. Приводятся точный алгоритм I для решения по частям обычной транспортной задачи линейного программирования (при $\left.M^{\prime}=\varnothing\right)$ и приближенный алгоритм II для решения полностью целочисленной задачи (при $M^{\prime} \equiv M$ ). Изучаются вопросы сходимости алгоритма I к оптимуму. Приводится экономическая интерпретация задачи. 